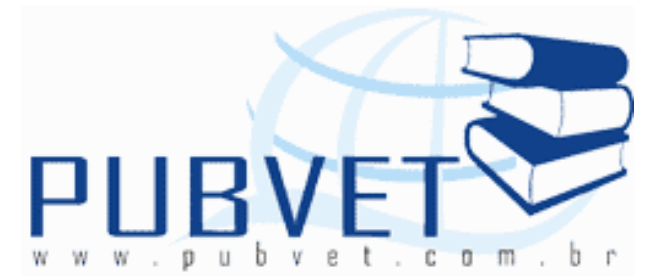

PUBVET, Publicações em Medicina Veterinária e Zootecnia.

\title{
Avaliação do bem estar animal durante o manejo pré abate e abate em um matadouro-frigorífico
}

Filippe Melo Diniz ${ }^{1}$, Laerte Pereira de Almeida ${ }^{2}$ e Garibalde Cândido Diniz ${ }^{3}$

${ }^{1}$ Discente Faculdade de Medicina Veterinária, Universidade Federal de Uberlândia.

${ }^{2}$ Docente Faculdade de Medicina Veterinária, Universidade Federal de Uberlândia.

${ }^{3}$ Agente de Inspeção Federal - Ministério da Agricultura, Pecuária e Abastecimento.

\section{Resumo}

Estudos sobre o Bem Estar Animal são de grande importância para a comunidade veterinária e científica, tendo em vista a pequena quantidade de trabalhos nessa área. Levando em conta que o Brasil é um dos maiores exportadores de carne bovina, suína e de aves, e que os países importadores desses produtos estão a exigir, cada vez mais, a utilização de práticas que respeitem o Bem Estar Animal, torna-se evidente a necessidade de se conhecer a realidade dos estabelecimentos de abate desses animais. Visando a obtenção de um padrão de qualidade sob o ponto de vista comercial e do abate humanitário. Neste sentido é que se propôs a realização de uma pesquisa com o objetivo de investigar os níveis de Bem Estar Animal, através da avaliação das práticas de manejo e abate em um Matadouro-Frigorífico em Uberlândia - 
DINIZ, F.M., ALMEIDA, L.P. e DINIZ, G.C. Avaliação do bem estar animal durante o manejo pré abate e abate em um matadouro-frigorífico. PUBVET, Londrina, V. 5, N. 24, Ed. 171, Art. $1152,2011$.

MG. Coletaram-se os dados através de um questionário padronizado em 7 visitas ao local, avaliando-se indicadores nas etapas de pré-abate e abate. Após a coleta, os dados foram processados e dispostos em tabelas. Para a análise, os dados foram transformados em indicadores e comparados com padrões da literatura. Essa comparação mostrou a presença de indicadores de Bem Estar Animal, entre os avaliados no Matadouro-Frigorífico pesquisado, em desacordo com os níveis de Bem Estar Animal aceitos. Conclui-se que a presença, no estabelecimento de abate avaliado, de indicadores de Bem Estar Animal fora dos padrões aceitáveis associa-se a alguns fatores como o manejo inadequado decorrente da falta de capacitação e nível de conhecimento insuficiente, sobre comportamento animal, por parte dos funcionários.

Palavras-chave: Bem Estar Animal; Matadouro - Frigorífico; Bovinos

\section{Evaluation of animal welfare during the handiling in an slaughterhouse}

\section{Abstract}

Studies on Animal Welfare have importance to veterinary and scientific community in view of the small amount of work in this area. Taking into account that Brazil is one of the largest exporters of beef, pork and chicken, and that importing countries are demanding these products, increasingly, the use of practices that respect the Animal Welfare, it is evident the need to know the reality of the slaughter establishments. Aiming to achieve a quality standard under the commercial point of view and humane slaughter. In this sense it is proposed to a survey in order to investigate the levels of Animal Welfare, through the evaluation of management practices and slaughter in a slaughter plant in Uberlandia - MG. Data were collected through a standardized questionnaire in seven site visits, evaluating indicators in the stages of preslaughter and slaughter. After collection, the data were processed and arranged in tables. For analysis, data were transformed into indicators and compared with the literature. This comparison showed the presence of indicators of Animal Welfare, among the subjects studied in the slaughter 
DINIZ, F.M., ALMEIDA, L.P. e DINIZ, G.C. Avaliação do bem estar animal durante o manejo pré abate e abate em um matadouro-frigorífico. PUBVET, Londrina, V. 5, N. 24, Ed. 171, Art. $1152,2011$.

plant, in violation of the Animal Welfare levels accepted. We conclude that the presence in the slaughterhouse assessed for indicators of Animal Welfare outside the acceptable standards is associated with factors such as inadequate management due to lack of training and insufficient level of knowledge about animal behavior, by employees.

Keywords: Animal welfare; slaughterhouse; bovine

\section{INTRODUÇÃO}

O processo de domesticação dos bovinos ocorreu a cerca de 6.000 anos. No início dessa relação, os bovinos eram beneficiados por uma maior abundância de alimentos e proteção, mas isso mudou com a revolução verde, na qual se iniciou a industrialização da pecuária, em que os animais passaram a ser criados para a produção de alimento em maior escala (MOLENTO; BOND, 2008). No entanto, com o passar do tempo, a importância da tecnologia do abate tornou-se motivo de preocupação para a ciência, pois se percebeu que o manejo dos animais antes do abate influenciava a qualidade da carne (SWATLAND, 2000).

A preocupação inicial com o Bem Estar dos animais surgiu a partir da exigência dos mercados internacionais, quando países importadores estabeleceram padrões e condições de criação e abate dos animais para a exportação (OLIVEIRA; BORTOLI; BARCELLOS, 2008). Neste sentido, desde o século passado, a prática de bons tratos aos animais é utilizada como um diferencial por muitos industriais para atrair clientes, conforme mostraram Carneiro et al. (1997) e Porter (1991).

A definição de Bem Estar Animal mais aceita atualmente é a de Broom (1986), ao afirmar que:

"Bem-Estar de um indivíduo é seu estado em relação às suas tentativas de se adaptar ao seu ambiente".

Segundo McGlone (2001), vale ressaltar que o conceito de Bem Estar Animal, deve estar vinculado ao de sustentabilidade, assegurando desta forma, 
DINIZ, F.M., ALMEIDA, L.P. e DINIZ, G.C. Avaliação do bem estar animal durante o manejo pré abate e abate em um matadouro-frigorífico. PUBVET, Londrina, V. 5, N. 24, Ed. 171, Art. $1152,2011$.

uma harmonia com o meio ambiente, com os animais, com os trabalhadores e com a comunidade, além de ser economicamente viável.

Para Broom e Molento (2004) o Bem Estar Animal é uma ciência que precisa estar sob o domínio de conhecimento de todos os profissionais que lidam com os animais, em qualquer parte da produção animal, de uma maneira tal que o treinamento, a capacitação e a sensibilidade dos funcionários dos frigoríficos são necessários, pois são eles que asseguram a não ocorrência, durante a permanência dos animais nos frigoríficos, de sofrimento desnecessário aos mesmos (ROÇA, 2001).

A importância do estudo sobre Bem Estar Animal está em garantir aos animais respeito e dignidade, assegurando assim bons níveis de Bem Estar Animal e melhores resultados econômicos. E diversas pesquisas demonstram que adotando-se medidas de manejo corretas, alcançamos melhores níveis de Bem Estar Animal (OLIVEIRA; BORTOLI; BARCELLOS, 2008).

Com o uso de práticas inadequadas de manejo, obtemos perdas de produção e isso foi demonstrado por Zapiola (2006) ao afirmar que uma auditoria americana estimou as perdas de $U \$ 47,00, U \$ 28,00$ e $U \$ 40,00$ nos anos de 1991, 1995 e 2005, respectivamente, para cada animal abatido somente por falhas de manejo. Esses valores representam um montante de $4 \%$ a $6 \%$ do valor do animal vivo. No Brasil, Almeida (2005) encontrou dados de perdas durante o toalete das regiões lesadas por falhas de manejo na ordem de 147 gramas por animal abatido.

O conhecimento sobre Bem Estar Animal associa-se com o conhecimento do comportamento dos animais. E, no caso dos bovinos, são animais rotineiros, dotados de uma boa memória e capazes de reconhecer seus tratadores e apresentar reações especificas a cada tipo de tratamento recebido (PARANHOS DA COSTA et al., 2002).

Para se melhorar os níveis de Bem Estar Animal é necessário adotar medidas como: Treinamento dos funcionários (OLIVEIRA; BORTOLI; BARCELLOS, 2008), instalações do abatedouro adequadas e bem planejadas, conforme demonstrado por Grandin (1980) e Van Putten e Elshoff (1978). 
DINIZ, F.M., ALMEIDA, L.P. e DINIZ, G.C. Avaliação do bem estar animal durante o manejo pré abate e abate em um matadouro-frigorífico. PUBVET, Londrina, V. 5, N. 24, Ed. 171, Art. $1152,2011$.

Com base nos fatos mencionados pode-se observar que o Bem Estar Animal é um tema de grande relevância e atualidade, que necessita de estudos regionais para que se possa compreender a realidade de estabelecimentos de abate de bovinos com fins de diagnóstico e intervenção visando à melhoria do estado atual do Bem Estar Animal nestes locais. A partir dessa necessidade é que se propôs a realização de uma pesquisa em um Matadouro-Frigorífico da região de Uberlândia-MG, buscando-se, através de indicadores, avaliar a situação do estabelecimento de abate de bovinos, comparando-a com padrões de BEA estabelecidos.

\section{MATERIAIS E MÉTODOS}

Essa pesquisa foi realizada em um Matadouro-Frigorífico na região de Uberlândia-MG, sob Inspeção Federal (S.I.F), durante o mês de julho e agosto de 2010.

A coleta de dados foi realizada por um único estudante do curso de Medicina Veterinária, previamente treinado. Avaliando-se a situação de Bem Estar Animal entre 600 bovinos durante a etapa de pré-abate e abate no Matadouro-Frigorífico. Coletaram-se, por meio de questionário padronizado (VOGEL; GRANDIN, 2009; BRASIL, 2000), dados referentes aos seguintes indicadores de Bem Estar Animal: Vocalizações, Quedas e Escorregões, Uso do Bastão Elétrico, Animais Sensíveis na Calha de Sangria, Arrastar Animais Sensíveis e Eficácia de Atordoamento.

Após a coleta, medidas de controle de qualidade dos dados foram aplicadas. E, uma vez verificada a ausência de erros os mesmos foram analisados e os resultados dispostos em tabelas e gráfico. Comparando os níveis dos indicadores encontrados com os níveis de padrões exigidos e aceitos para o estado de Bem Estar Animal. 


\section{RESULTADOS E DISCUSSÃO}

Tabela 1 - Indicadores de Bem Estar Animal (BEA), resultado obtido em matadouro-frigorífico de bovinos. Uberlândia-MG, 2010.

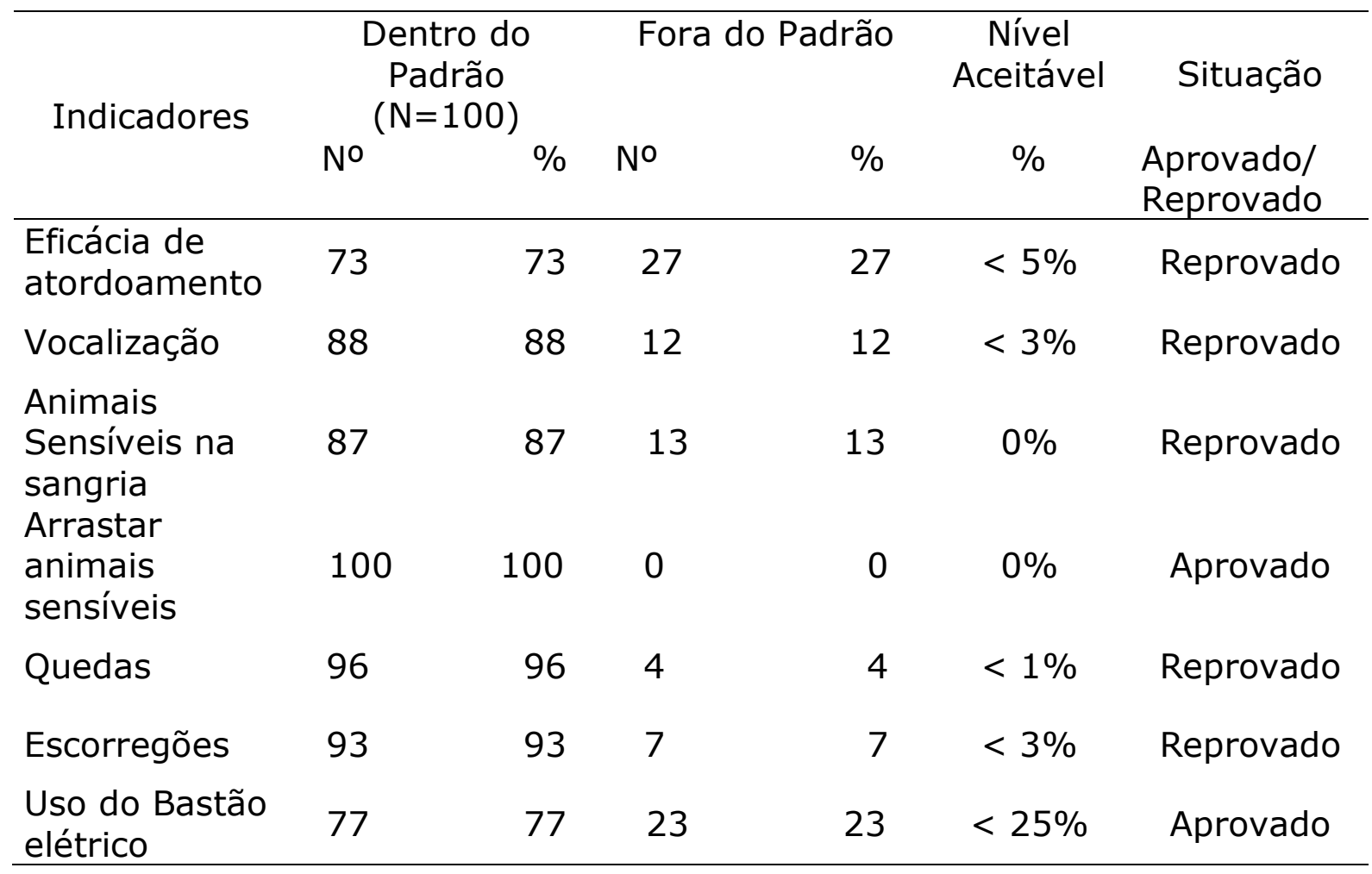

Primeiramente, podemos notar que de acordo com a Tabela 1, do total de 7 indicadores de Bem Estar Animal, somente 2 (28,5\%) apresentaram os resultados de acordo com o padrão aceitável. Isso nos mostra a realidade dos níveis de Bem Estar Animal encontrados no estabelecimento avaliado. Sugerindo a possibilidade de aperfeiçoamento de algumas condições, necessárias para se alcançar os padrões aceitáveis de Bem Estar Animal em diversos itens.

Uma possível causa para essa freqüência de indicadores fora dos níveis aceitáveis foi apontada por Grandin (1996) e Cruz; Souza (2005) ao demonstrarem que os níveis de Bem Estar Animal estão relacionados com as instalações do estabelecimento de abate e outros itens, como a segurança para 
os operadores. Porém, ao analisarmos as instalações do estabelecimento em estudo, observamos que o mesmo possui "boas instalações", exceto no caso do Box de contenção, o qual não realizava satisfatoriamente sua função desejada. Acreditamos que as principais falhas são a falta de treinamento, seguida pelo uso do bastão elétrico de maneira incorreta, falta de contenção dos animais e carência de funcionários.

Com relação ao item "Uso do Bastão Elétrico", Tabela 1, nota-se que o mesmo está de acordo com os níveis aceitos de Bem Estar Animal, sendo feito uma ressalva de que, a metodologia utilizada para a avaliação do presente item, foi a de que deveria marcar os animais que vocalizaram em decorrência do uso do bastão elétrico ou se o mesmo era utilizado em áreas sensíveis do animal, que são: "face, olhos, orelhas, nariz, úbere, testículos e região anal e genital dos animais" (VOGEL; GRANDIN, 2009).

Grandin (2007), durante a analise de 44 plantas frigorificas, encontrou um resultado no qual, 1 planta (2\%), apresentou $44 \%$ de animais manejados com o uso do bastão elétrico, o que está fora dos padrões aceitos. Por outro lado, Vogel; Grandin (2009), ao analisarem 26 plantas frigorificas, não encontraram nenhuma delas com o uso do bastão elétrico fora dos limites permitidos. No presente estudo, observou-se que alguns servidores não vinculados especificamente à função de manejar os animais utilizavam exageradamente o bastão elétrico na tentativa de auxiliarem os servidores dessa função. 
DINIZ, F.M., ALMEIDA, L.P. e DINIZ, G.C. Avaliação do bem estar animal durante o manejo pré abate e abate em um matadouro-frigorífico. PUBVET, Londrina, V. 5, N. 24, Ed. 171, Art. $1152,2011$.

Tabela 2- Eficácia de atordoamento, resultado obtido em matadouro-frigorífico de bovinos. Uberlândia-MG, 2010.

Número de Tiros Efetuados
Número de Animais $(n=100)$
Atordoamento completo $\mathrm{c} / 1$ tiro Atordoamento completo $\mathrm{c} / 2$ tiros Atordoamento completo $\mathrm{c} / 3$ tiros
73

23

04
Porcentagem

Segundo Gregory, Lee e Widdicombe (2007), o atordoamento tem como objetivo, a insensibilidade do animal, para que este não sofra dor durante a etapa de sangria. Devemos ressaltar que o método de insensibilização utilizado é a pistola pneumática de dardo cativo. Uma vez que o nível aceito de animais atordoados com mais de uma tentativa é de 5\% (Vogel e Grandin, 2009), percebe-se que a situação do Matadouro-Frigorífico avaliado é possível de melhora, uma vez que em $27 \%$ dos casos foram necessárias mais de uma tentativa para o atordoamento dos animais (Tabela 1). Da mesma maneira, Almeida (2005) em um estudo, encontrou que do total de 800 animais abatidos, $186(23,25 \%)$ só foram atordoados com mais de uma tentativa.

Durante o presente estudo foi constatado um número insuficiente de funcionários responsáveis pela função de manejar os animais, atordoá-los e colocá-los nos trilhos, o que gera uma exaustão física e mental, contribuindo para um maior uso do bastão elétrico e mais falhas de atordoamento. Outro fato que chamou a atenção e que contribuiu para maior dificuldade de atordoamento dos animais se refere à contenção incompleta do animal no Box de Atordoamento, possibilitando a movimentação do animal no local, dificultando a insensibilização do mesmo. 
DINIZ, F.M., ALMEIDA, L.P. e DINIZ, G.C. Avaliação do bem estar animal durante o manejo pré abate e abate em um matadouro-frigorífico. PUBVET, Londrina, V. 5, N. 24, Ed. 171, Art. $1152,2011$.

Tabela 3 - Animais sensíveis na calha de sangria, resultado obtido em matadouro-frigorifico de bovinos. Uberlândia - MG, 2010.

\begin{tabular}{lcc}
\hline Condição do Animal & $\begin{array}{c}\text { Número } \\
(\mathrm{n}=100)\end{array}$ & Porcentagem \\
\hline Animais completamente insensibilizados & 87 & 87 \\
Animais com olhos movendo quando tocados (1) & 0 & 0 \\
Animais piscando (2) & 0 & 0 \\
Animais com Respiração Ritmada (3) & 0 & 0 \\
Animais vocalizando (4) & 0 & 0 \\
Animais com reflexo de equilíbrio (5) & 11 & 11 \\
Animais com (3) e (5) simultaneamente & 1 & 1 \\
Animais com (1), (2) e (4) simultaneamente & 1 & 1 \\
\hline
\end{tabular}

Grandin (2007), afirma que os níveis aceitos de animais sensíveis na calha de sangria são de $0 \%$, ou seja, nenhum animal pode expressar comportamento de dor ou sensibilidade e, qualquer resultado maior do que $0 \%$ é considerado reprovado. Essa autora ao realizar um estudo que avaliou um total de 44 plantas frigoríficas, obteve resultados que mostraram que em 43 delas (98\%), os animais estavam $100 \%$ insensibilizados e em 1 planta (2\%), menos do que $100 \%$ dos animais estavam insensibilizados, sendo assim reprovado no estudo.

Ainda com relação a esse item, Neves (2008) não constatou nenhum animal sensível na calha de sangria no abate efetuado com pistola de dardo cativo com penetração. No entanto, Almeida (2005), ao analisar 800 animais, encontrou que desses, 49 animais $(6,13 \%)$ estavam sensíveis e 5 animais $(10,5 \%)$ apresentaram respiração rítmica e 44 a correção de postura $(89,8 \%)$. 
DINIZ, F.M., ALMEIDA, L.P. e DINIZ, G.C. Avaliação do bem estar animal durante o manejo pré abate e abate em um matadouro-frigorífico. PUBVET, Londrina, V. 5, N. 24, Ed. 171, Art. $1152,2011$.

Tabela 4 - Causa de vocalização dos animais, resultado obtido em matadourofrigorífico de bovinos. Uberlândia- MG, 2010.

\begin{tabular}{lcc}
\hline & $\begin{array}{c}\text { Número de Animais } \\
(\mathrm{n}=100)\end{array}$ & $\begin{array}{c}\text { Porcentagem } \\
\text { Causa de Vocalização }\end{array}$ \\
\hline Não Vocalização & 88 & 88 \\
Vocalização devido a Bastão Elétrico & 10 & 10 \\
Vocalização devido a Quedas/Escorregões & 1 & 1 \\
Vocalização por motivo não identificado & 1 & 1 \\
\hline
\end{tabular}

Segundo Vogel; Grandin (2009) o nível aceito de vocalização é de até $3 \%$ dos animais. Dessa maneira, observamos que, no presente estudo, $12 \%$ dos animais vocalizaram o que se mostra fora do limite aceitável pelos pesquisadores mencionados.

Um fato que chamou a atenção durante a avaliação desse item foi a presença de indivíduos não vinculados a atividade de manejar os animais, tais como proprietários e outros servidores, com o intuito de auxiliar as pessoas responsáveis pela tarefa, usavam $\mathrm{o}$ bastão elétrico excessivamente e desnecessariamente nos animais para conduzi-los para o Box de contenção. Observou-se, ainda que, por várias vezes, os bastões elétricos foram usados repetidamente, e, até em momentos desnecessários. Uma das possíveis medidas para reduzir tanto o uso do bastão elétrico, quanto às vocalizações, seria providenciar uma melhor iluminação no Box de atordoamento, bem como o uso de bandeiras para tocar os animais, além de treinamento com os servidores responsáveis por essa tarefa.

A título de comparação, Almeida (2005) ao analisar 800 animais, verificou que 207 deles (25,88\%) apresentaram vocalização. Desse total, 175 $(84,54 \%)$ foram causadas pelo uso de choque elétrico, enquanto que 13 $(6,28 \%)$ ocorreu durante o atordoamento, $1(0,48 \%)$ por quedas e $18(8,70 \%)$ decorrente de pressão na contenção do animal.

Com relação aos itens relacionados com o uso do "Bastão Elétrico", Tabela 1 e Tabela 4, podemos observar que em 23\% dos casos foi utilizado este instrumento e $10 \%$ de animais vocalizaram após uso do bastão elétrico, 
DINIZ, F.M., ALMEIDA, L.P. e DINIZ, G.C. Avaliação do bem estar animal durante o manejo pré abate e abate em um matadouro-frigorífico. PUBVET, Londrina, V. 5, N. 24, Ed. 171, Art. $1152,2011$.

respectivamente. Ao voltamos a metodologia aplicada no estudo, podemos perceber que no item "Uso do bastão elétrico" era para ser marcado quando ocorria vocalizações ou o mesmo era utilizado em áreas sensíveis dos animais. Dessa forma, poderíamos somar os resultados encontrados na Tabela 1 e 4 para "Bastão elétrico", obtendo-se um resultado de 33\%, o qual está em desacordo com o preconizado na literatura - $25 \%$ de uso do bastão elétrico (VOGEL E GRANDIN, 2009) -.

De acordo com a metodologia proposta por Grandin (2007) e Vogel e Grandin (2009), os resultados encontrados nessa pesquisa, estão demonstrando que são necessárias medidas corretivas para melhorar os indicadores de Bem Estar Animal.

\section{CONCLUSÕES}

A partir da metodologia utilizada e com base nos resultados dos indicadores obtidos e comparados com os padrões de Bem Estar Animal, este estudo permite concluir que a situação do estabelecimento de abate avaliado pode ser melhorada, para que se possa conseguir atingir os níveis desejados para o Bem estar Animal. Com conseqüências para a qualidade da carne e reduzindo o estresse de animais e homens.

Entre as sugestões possíveis de serem aplicadas para a melhora do estabelecimento de abate avaliado estão:

- Oferecer treinamento para os servidores responsáveis pelas etapas de pré-abate e abate dos animais, principalmente voltado aos tipos de comportamento animal, o que facilitaria o manejo dos mesmos.

- Iniciar a utilização de bandeiras coloridas para manejar os animais, aproveitando o campo de visão dos mesmos para facilitar o manejo, deixando o uso do bastão elétrico somente para situações extremas. 
DINIZ, F.M., ALMEIDA, L.P. e DINIZ, G.C. Avaliação do bem estar animal durante o manejo pré abate e abate em um matadouro-frigorífico. PUBVET, Londrina, V. 5, N. 24, Ed. 171, Art. $1152,2011$.

- Colocação de animais em lotes menores, de uma maneira tal que não acumule mais do que 5 animais na seringa, evitando assim quedas e escorregões desnecessários.

- Criação de mecanismo, no Box de atordoamento, que possibilite ao animal permanecer imóvel durante o atordoamento, evitando a repetição de disparos com a pistola pneumática. Neste mesmo local, a adaptação de algumas condições de trabalho poderia reduzir a presença de barulho, facilitando o atordoamento dos animais.

- Contratação de um servidor, para que fiquem 3 indivíduos responsáveis pelas funções de pré-abate e abate dos animais, realizando um rodízio entre eles de uma forma tal, que todos passem por todas as etapas, ou seja, um fica manejando os animais, outro atordoando e outro suspendendo os mesmos.

Por fim, acreditamos que somente a partir da produção de informações sobre o Bem Estar Animal e especificidades do comportamento dos animais e sua transmissão aos indivíduos que trabalham cotidianamente com essas atividades é que poderemos conseguir maior conscientização para a necessidade de se respeitar o Bem Estar Animal e se promover mudanças capazes de alterar os indicadores segundo os padrões de Bem Estar Animal.

\section{REFERÊNCIAS BIBLIGRÁFICAS}

MOLENTO, C. F. M.; BOND, G. B. Produção e Bem Estar Animal: aspectos éticos e técnicos da produção de bovinos. Ciência Veterinária Nos Trópicos, Recife, v. 11, n. 1, p.36-42, 01 abr. 2008.

SWATLAND,H.J. Slaughtering. Disponível em:

<http://www.bert.aps.uoguelph.ca/ swatland/ch1.9.htm>. 2000. 10p. Acesso em: 08 Abr.2010. 
OLIVEIRA, C. B. de; BORTOLI, E. C. de; BARCELLOS, J. O. J.. Diferenciação por qualidade da carne bovina: a ótica do bem-estar animal. Ciência Rural, Santa Maria, v. 7, n. 38, p.20922096, 01 out. 2008.

CARNEIRO, J.M.T. et al. Porter revisitado: análise crítica da tipologia estratégica do mestre. In: ENANPAD, 21., 1997, Rio de Janeiro. Anais... Rio de Janeiro, 1997.

PORTER, M.E. Estratégia competitiva: Técnicas para análise de indústrias e da concorrência. 7.ed. Rio de Janeiro: Campus, 1991.

BROOM, D.M. Indicators of poor welfare. British Veterinary Journal, London, v.142, p.524526,1986

McGLONE, J.J. Farm animal welfare in the context of other society issues: toward sustainable systems. Livestock Production Science, v.72, p.75-81,2001.

BROOM, D.M.; MOLENTO, C.F.M. Bem-estar animal: conceito e questões relacionadas Revisão. Archives of Veterinary Science v.9, n.2, p.1-11, 2004.

ROÇA, R. de O. Abate humanitário: manejo Ante-mortem. Revista Tec Carnes, Campinas, v. 3, n. 1, p.7-12, 2001.

ZAPIOLA, M.G. El bienestar animal y la calidad de la carne. In: . Bienestar animal y calidad de la carne. Argentina: Instituto de Promoción de la Carne Vacuna Argentina, IPCVA, 2006. (Cuadernillo Técnico)

ALMEIDA, L. A. M. Manejo no pré-abate de bovinos: aspectos comportamentais e perdas econômicas por contusõeS. 2005. 75 f. Dissertação (Mestrado) - Curso de Medicina Veterinária, Departamento de Faculdade de Ciências Agrárias e Veterinárias, Unesp, Jaboticabal, 2005. Disponível em:

<http://www.biblioteca.unesp.br/bibliotecadigital/document/?did=3362>. Acesso em: 05 ago. 2010.

PARANHOS DA COSTA, M.J.R. . Ambiência e qualidade de carne. In: L.A. Josahkian (ed.) Anais... Uberaba, pp. 170-174, 2002.

GRANDIN, T. Observations of cattle behaviour applied to the design of cattle handling facilities. Applied Animal Behavioural Science. v.6 n. 19. 1980.

VAN PUTTEN,G. e ELSHOF,W.J. Observations on the effect of transport on the well being and lean quality of pigs. Animal Regulatory Studies. v.1, p.:247-271, 1978.

VOGEL, Kurt; GRANDIN, Temple. 2009 Restaurant Animal Welfare and Humane Slaughter Audits in Federally Inspected Beef and Pork Slaughter Plants in the U.S. Disponível em: <http://www.grandin.com/survey/2009. restaurant.audits.html>. Acesso em: 30 nov. 2010.

BRASIL. Ministério da Agricultura Instrução normativa n03 de 17 de janeiro de 200. Diário Oficial da União, Brasília, DF, 24 jan. 2000. Seção, 1 p. 14. Disponível em: <http://extranet.agricultura.gov.br/sislegis-consulta/consultarLegislacao.do?operacao $=$ visualizar\&id $=1793>$. Acesso em: 24 mar. 2010.

GRANDIN, T. Factors that impede animal movement at slaughter plants. Journal of American Veterinary Medical Association, Schaumburg, v.209, n.4, p.757-759, 1996b. 
CRUZ, V.F.; SOUSA, P. Sistema integrado de monitoramento do bem-estar animal. EMBRAPA Suínos e Aves. Artigos. 2005.

GRANDIN, Temple. 2007 Restaurant Animal Welfare and Humane Slaughter Audits in Federally Inspected Beef and Pork Slaughter Plants in the U.S. and Canada. Disponível em: <http://www.grandin.com/survey/2007.restaurant.audits.html>. Acesso em: 28 out. 2010.

GREGORY, N., LEE, C.J., WIDDICOMBE, J.P. Depth of concussion in cattle shot by penetrating captive bolt. Meat Science 77, 499-503. 2007a.

NEVES, J. E. G. Influências de métodos de abate no bem-estar e na qualidade da carne de bovinos. 2008. 69 f. Dissertação (Mestrado) - Curso de Medicina Veterinária, Departamento de Faculdade de Ciências Agrárias e Veterinárias, Unesp, Jaboticabal, 2008. Disponível em: <http://www.fcav.unesp.br/download/pgtrabs/zoo/m/3486.pdf>. Acesso em: 5 ago. 2010. 